Document downloaded from:

http://hdl.handle.net/10251/176110

This paper must be cited as:

Bonet Solves, JA.; Ricker, WJ. (2020). Order spectrum of the Cesàro operator in Banach lattice sequence spaces. Positivity. 24(3):593-603. https://doi.org/10.1007/s11117-01900699-9

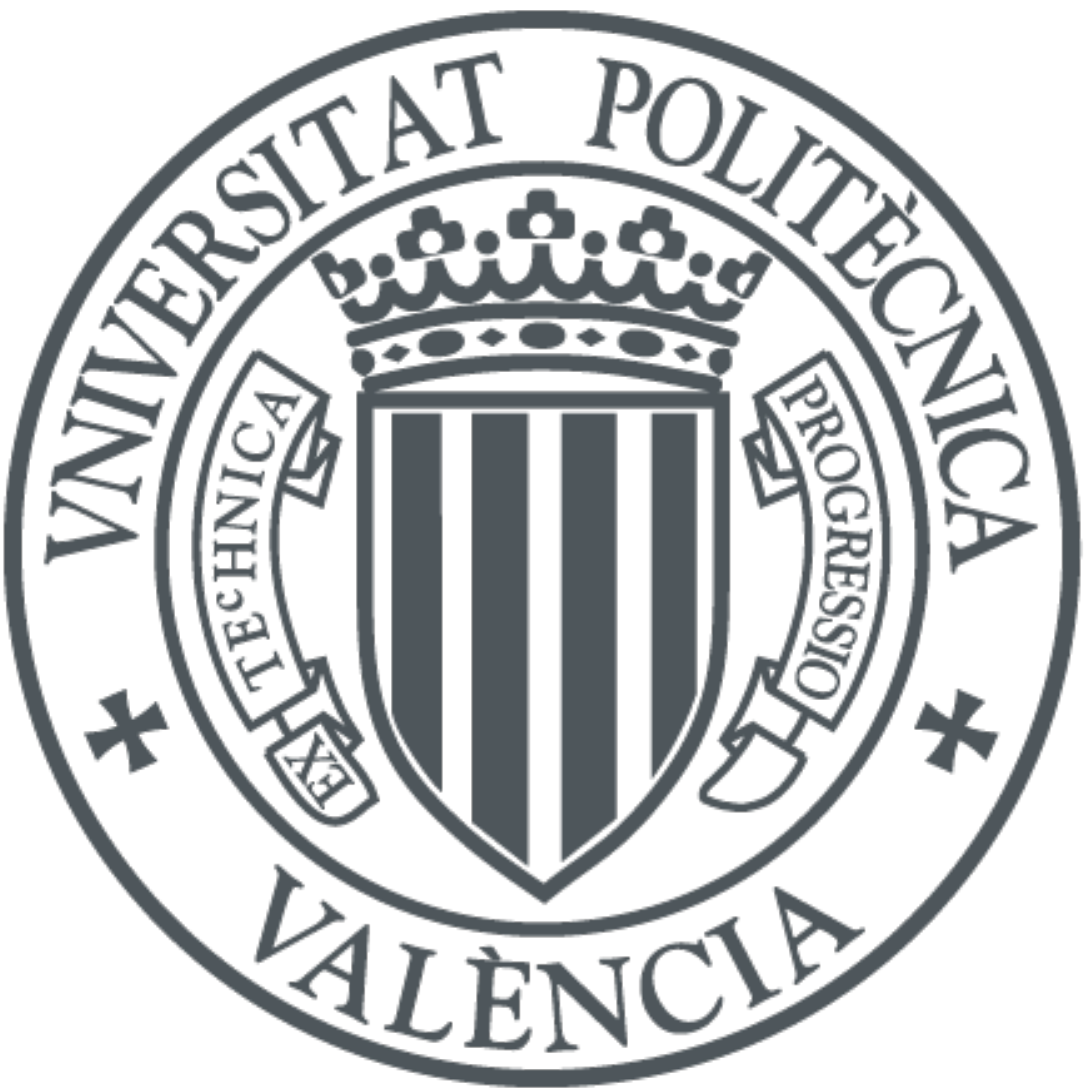

The final publication is available at

https://doi.org/10.1007/s11117-019-00699-9

Copyright Springer-Verlag

Additional Information 


\title{
ORDER SPECTRUM OF THE CESÀRO OPERATOR IN BANACH LATTICE SEQUENCE SPACES
}

\author{
J. BONET AND W.J. RICKER
}

\begin{abstract}
The discrete Cesàro operator $C$ acts continuously in various classical Banach sequence spaces within $\mathbb{C}^{\mathbb{N}}$. For the coordinatewise order, many such sequence spaces $X$ are also complex Banach lattices (eg. $c_{0}, \ell^{p}$ for $1<p \leq \infty$, and $\operatorname{ces}(p)$ for $p \in\{0\} \cup(1, \infty))$. In such Banach lattice sequence spaces, $C$ is always a positive operator. Hence, its order spectrum is well defined within the Banach algebra of all regular operators on $X$. The purpose of this note is to show, for every $X$ belonging to the above list of Banach lattice sequence spaces, that the order spectrum $\sigma_{\mathrm{o}}(C)$ of $C$ coincides with its usual spectrum $\sigma(C)$ when $C$ is considered as a continuous linear operator on the Banach space $X$.
\end{abstract}

\section{INTRODUCTION}

Let $E$ be a complex Banach lattice and $\mathcal{L}(E)$ denote the unital Banach algebra of all continuous linear operators from $E$ into itself, equipped with the operator norm $\|\cdot\|_{\text {op }}$. The unit is the identity operator $I: E \longrightarrow E$. Associated with each $T \in \mathcal{L}(E)$ is its spectrum

$$
\sigma(T):=\{\lambda \in \mathbb{C}:(\lambda I-T) \text { is not invertible in } \mathcal{L}(E)\}
$$

and its resolvent set $\rho(T):=\mathbb{C} \backslash \sigma(T)$. An operator $T \in \mathcal{L}(E)$ is called regular if it is a finite linear combination of positive operators. The complex vector space of all regular operators is denoted by $\mathcal{L}^{r}(E)$; it is also a unital Banach algebra for the norm

$$
\|T\|_{r}:=\inf \left\{\|S\|_{\text {op }}: S \in \mathcal{L}(E), S \geq 0,|T(z)| \leq S(|z|) \forall z \in E\right\}, \quad T \in \mathcal{L}^{r}(E) .
$$

Again $I: E \longrightarrow E$ is the unit. Moreover, $\|T\|_{\text {op }} \leq\|T\|_{r}$ for $T \in \mathcal{L}^{r}(E)$, with equality whenever $T \geq 0$ (i.e., if $T$ is a positive operator). The spectrum of $T \in \mathcal{L}^{r}(E)$, considered as an element of the Banach algebra $\mathcal{L}^{r}(E)$, is denoted by $\sigma_{\mathrm{o}}(T)$ and is called its order spectrum. Then $\rho_{\mathrm{o}}(T):=\mathbb{C} \backslash \sigma_{\mathrm{o}}(T)$ is the order resolvent of $T$. Clearly

$$
\sigma(T) \subseteq \sigma_{\mathrm{o}}(T), \quad T \in \mathcal{L}^{r}(E) .
$$

From the usual formula for the spectral radius, [5, Ch.I, §2, Proposition 8], it follows that the spectral radii for $T \in \mathcal{L}^{r}(E)$ satisfy $r(T)=r_{\mathrm{o}}(T)$ whenever $T \geq 0$. Standard references for the above concepts and facts are [3], [17], [18], for example.

It is clear from (1.2) that $r(T) \leq r_{\mathrm{o}}(T)$ for $T \in \mathcal{L}^{r}(E)$. So, if $r(T)<r_{\mathrm{o}}(T)$, then (1.2) cannot be an equality. This is the strategy applied in [18, pp.79-80] to exhibit a regular operator for which $\sigma(T) \varsubsetneqq \sigma_{\mathrm{o}}(T)$. For an example of a positive operator $T$ satisfying $\sigma(T) \varsubsetneqq \sigma_{\mathrm{o}}(T)$, see [3, pp.283-284]. In the contrary direction, a rich supply of classical operators $T$ for which the equality

$$
\sigma(T)=\sigma_{\mathrm{o}}(T)
$$

2010 Mathematics Subject Classification. Primary 47A10, 47B37, 47B65, 47L10; Secondary 46A45, 46B45, 47C05.

Key words and phrases. Banach algebra, Banach sequence space, Cesàro operator, spectrum, order spectrum. 
is satisfied arise in harmonic analysis, [3, Theorem 3.4].

The aim of this note is to contribute two further classes of operators $T$ which satisfy (1.3). In Section 2 it is shown that in any Banach function space $E$, all multiplication operators $T$ by $L^{\infty}$-functions are regular operators and satisfy (1.3). This is a consequence of the fact that the algebra of such multiplication operators is maximal commutative. Let $\mathbb{N}:=\{1,2, \ldots\}$. The remaining three sections deal with the classical Cesàro operator $C: \mathbb{C}^{\mathbb{N}} \longrightarrow \mathbb{C}^{\mathbb{N}}$ defined by

$$
C(x):=\left(\frac{1}{n} \sum_{k=1}^{n} x_{k}\right)_{n=1}^{\infty} \quad x=\left(x_{n}\right)_{n=1}^{\infty} \in \mathbb{C}^{\mathbb{N}},
$$

which is clearly a positive operator for the coordinatewise order in the positive cone of $\mathbb{C}^{\mathbb{N}}=\mathbb{R}^{\mathbb{N}} \oplus i \mathbb{R}^{\mathbb{N}}$. Section 3 establishes some general results for determining the regularity of linear operators in Banach lattice sequence spaces. These results are designed to apply to the particular operators $(C-\lambda I)^{-1}$, where $C$ is given in (1.4). In Section 4 we will consider the restriction of $C$ to the Banach lattice sequence spaces $c_{0}$ and $\ell^{p}, 1<p \leq \infty$, and show that (1.3) is satisfied in all cases (with $C$ in place of $T$ ). Section 5 is devoted to proving the same fact, but now when $C$ acts in the discrete Cesàro spaces $\operatorname{ces}(p), 1<p<\infty$, and in $\operatorname{ces}(0)$.

\section{Multiplication Operators}

Let $(\Omega, \Sigma, \mu)$ be a localizable measure space (in the sense of [10, 64A]), that is, the associated measure algebra is a complete Boolean algebra and, for every measurable set $A \in \Sigma$ with $\mu(A)>0$ there exists $B \in \Sigma$ such that $B \subseteq A$ and $0<\mu(B)<\infty$ (i.e., $\mu$ has the finite subset property). All $\sigma$-finite measures are localizable, [10, 64H Proposition]. Every Banach function space $E$ (of $\mathbb{C}$-valued functions) over $(\Omega, \Sigma, \mu)$ is a complex Banach lattice for the pointwise $\mu$-a.e. order. Given any $\varphi \in L^{\infty}(\mu)$, the multiplication operator $M_{\varphi}: E \longrightarrow E$ defined by $f \longmapsto \varphi f$, for $f \in E$, belongs to $\mathcal{L}(E)$ and satisfies $\left\|M_{\varphi}\right\|_{\text {op }}=\|\varphi\|_{\infty}$. Define a unital, commutative subalgebra of $\mathcal{L}(E)$ by

$$
\mathcal{M}_{E}\left(L^{\infty}(\mu)\right):=\left\{M_{\varphi}: \varphi \in L^{\infty}(\mu)\right\}
$$

the unit is the identity operator $I=M_{1}$ where 1 is the constant function 1 on $\Omega$. Recall that the commutant of $\mathcal{M}_{E}\left(L^{\infty}(\mu)\right)$ is defined by

$$
\mathcal{M}_{E}\left(L^{\infty}(\mu)\right)^{c}:=\left\{A \in \mathcal{L}(E): A M_{\varphi}=M_{\varphi} A \forall \varphi \in L^{\infty}(\mu)\right\} \subseteq \mathcal{L}(E) .
$$

It is known that $\mathcal{M}_{E}\left(L^{\infty}(\mu)\right)$ is a maximal commutatitive, unital subalgebra of $\mathcal{L}(E)$, that is, $\mathcal{M}_{E}\left(L^{\infty}(\mu)\right)=\mathcal{M}_{E}\left(L^{\infty}(\mu)\right)^{c}$, [9, Proposition 2.2]. Moreover, also the bicommutant $\mathcal{M}_{E}\left(L^{\infty}(\mu)\right)^{c c}=\mathcal{M}_{E}\left(L^{\infty}(\mu)\right)$.

Proposition 2.1. Let $(\Omega, \Sigma, \mu)$ be a localizable measure space and $E$ be a Banach function space over $(\Omega, \Sigma, \mu)$.

(i) $\mathcal{M}_{E}\left(L^{\infty}(\mu)\right) \subseteq \mathcal{L}^{r}(E)$.

(ii) $\mathcal{M}_{E}\left(L^{\infty}(\mu)\right)$ is inverse closed in $\mathcal{L}(E)$. That is, if $T \in \mathcal{M}_{E}\left(L^{\infty}(\mu)\right)$ is invertible in $\mathcal{L}(E)$ (i.e., there exists $S \in \mathcal{L}(E)$ satisfying $S T=I=T S)$, then necessarily $S \in \mathcal{M}_{E}\left(L^{\infty}(\mu)\right)$.

(iii) For every $T \in \mathcal{M}_{E}\left(L^{\infty}(\mu)\right)$ we have $\sigma_{\mathrm{o}}(T)=\sigma(T)$.

Proof. (i) Let $\varphi \in L^{\infty}(\mu)$. Then $\varphi=\left[(\operatorname{Re} \varphi)^{+}-(\operatorname{Re} \varphi)^{-}\right]+i\left[(\operatorname{Im} \varphi)^{+}-(\operatorname{Im} \varphi)^{-}\right]$with all four functions $(\operatorname{Re} \varphi)^{+},(\operatorname{Re} \varphi)^{-},(\operatorname{Im} \varphi)^{+},(\operatorname{Im} \varphi)^{-}$belonging to the positive cone $L^{\infty}(\mu)^{+}$ of $L^{\infty}(\mu)$. Since $M_{\varphi}=\left[M_{(\operatorname{Re} \varphi)^{+}}-M_{(\operatorname{Re} \varphi)^{-}}\right]+i\left[M_{(\operatorname{Im} \varphi)^{+}}-M_{(\operatorname{Im} \varphi)^{-}}\right]$is a linear combination of positive operators, it is clear that $M_{\varphi} \in \mathcal{L}^{r}(E)$. 
(ii) Since $\mathcal{M}_{E}\left(L^{\infty}(\mu)\right)$ is maximal commutative in $\mathcal{L}(E)$, it follows that $\mathcal{M}_{E}\left(L^{\infty}(\mu)\right)$ is inverse closed in $\mathcal{L}(E)$, [5, Ch.II, §15, Theorem 4].

(iii) In view of (1.1) it suffices to show that $\rho(T) \subseteq \rho_{\mathrm{o}}(T)$. Suppose that $T=M_{\varphi}$ with $\varphi \in L^{\infty}(\mu)$. Fix $\lambda \in \rho(T)$. Then $\lambda I-T=M_{(\lambda \mathbf{1}-\varphi)}$ belongs to $\mathcal{M}_{E}\left(L^{\infty}(\mu)\right)$ because $(\lambda \mathbf{1}-\varphi) \in L^{\infty}(\mu)$. Since $M_{(\lambda \mathbf{1}-\varphi)}$ is invertible in $\mathcal{L}(E)$, it follows from part (ii) that actually $(\lambda I-T)^{-1} \in \mathcal{M}_{E}\left(L^{\infty}(\mu)\right)$ and hence, by part (i), that also $(\lambda I-T)^{-1} \in$ $\mathcal{L}^{r}(E)$.

Remark 2.2. We point out that $\|T\|_{\text {op }}=\|T\|_{r}$ for each $T \in \mathcal{M}_{E}\left(L^{\infty}(\mu)\right)$. Indeed, let $\varphi \in L^{\infty}(\mu)$ satisfy $T=M_{\varphi}$, in which case $\left\|M_{\varphi}\right\|_{\text {op }}=\|\varphi\|_{\infty}$. Define $S:=\|\varphi\|_{\infty} I$ and note that $S \geq 0$ with $\|S\|_{\text {op }}=\|\varphi\|_{\infty}$. Moreover,

$$
\left|M_{\varphi}(f)\right|=|\varphi f| \leq\|\varphi\|_{\infty}|f|=S(|f|), \quad f \in E,
$$

and so $\|T\|_{r} \leq\|S\|_{\text {op }}=\|\varphi\|_{\infty}=\|T\|_{\text {op }}$; see (1.1). The reverse inequality $\|T\|_{\text {op }} \leq\|T\|_{r}$ always holds.

\section{The Cesàro operator in Banach sequence spaces}

We begin with some preliminaries. Equipped with the topology of pointwise convergence $\mathbb{C}^{\mathbb{N}}$ is a locally convex Fréchet space. Let $A=\left(a_{n m}\right)_{n, m=1}^{\infty}$ be any lower triangular (infinite) matrix, i.e., $a_{n m}=0$ whenever $m>n$. Then $A$ induces the continuous linear operator $T_{A}: \mathbb{C}^{\mathbb{N}} \longrightarrow \mathbb{C}^{\mathbb{N}}$ defined by

$$
T_{A}(x):=\left(\sum_{m=1}^{\infty} a_{n m} x_{m}\right)_{n=1}^{\infty}, \quad x \in \mathbb{C}^{\mathbb{N}} .
$$

For $x \in \mathbb{C}^{\mathbb{N}}$ define $|x|:=\left(\left|x_{n}\right|\right)_{n=1}^{\infty}$. Then also $|x| \in \mathbb{C}^{\mathbb{N}}$. A vector subspace $X \subseteq \mathbb{C}^{\mathbb{N}}$ is called solid (or an ideal) if $y \in X$ whenever $x \in X$ and $y \in \mathbb{C}^{\mathbb{N}}$ satisfy $|y| \leq|x|$. It is always assumed that $X$ contains the vector space consisting of all elements of $\mathbb{C}^{\mathbb{N}}$ which have only finitely many non-zero coordinates. In addition, it is assumed that $X$ has a norm $\|\cdot\|_{X}$ with respect to which it is a complex Banach lattice for the coordinatewise order and such that the natural inclusion $X \subseteq \mathbb{C}^{\mathbb{N}}$ is continuous. Under the previous requirements $X$ is called a Banach lattice sequence space.

Lemma 3.1. Let $A=\left(a_{n m}\right)_{n, m=1}^{\infty}$ be a lower triangular matrix with all entries nonnegative real numbers and $X \subseteq \mathbb{C}^{\mathbb{N}}$ be a Banach lattice sequence space such that $T_{A}(X) \subseteq$ $X$. Let $B=\left(b_{n m}\right)_{n, m=1}^{\infty}$ be any matrix such that

$$
\left|b_{n m}\right| \leq a_{n m}, \quad n, m \in \mathbb{N} .
$$

Then the restricted operator $T_{A}: X \longrightarrow X$ belongs to $\mathcal{L}(X)$. Moreover, $T_{B}: \mathbb{C}^{\mathbb{N}} \longrightarrow \mathbb{C}^{\mathbb{N}}$ satisfies $T_{B}(X) \subseteq X$ and the restricted operator $T_{B}: X \longrightarrow X$ also belongs to $\mathcal{L}(X)$. In addition, $\left\|T_{B}\right\|_{\mathrm{op}} \leq\left\|T_{A}\right\|_{\mathrm{op}}$.

Proof. Condition (3.2) implies that $B$ is also a lower triangular matrix. Moreover, the continuity of both $T_{A}: \mathbb{C}^{\mathbb{N}} \longrightarrow \mathbb{C}^{\mathbb{N}}$ and of the inclusion map $X \subseteq \mathbb{C}^{\mathbb{N}}$ imply, via the Closed Graph Theorem in the Banach space $X$, that the restricted operator $T_{A} \in \mathcal{L}(X)$.

Given $x \in X$ we have for each $n \in \mathbb{N}$, via (3.2), that

$$
\left(T_{B}(x)\right)_{n}=\left|\sum_{m=1}^{\infty} b_{n m} x_{m}\right| \leq \sum_{m=1}^{\infty}\left|b_{n m}\right| \cdot\left|x_{m}\right| \leq \sum_{m=1}^{\infty} a_{n m}\left|x_{m}\right|=\left(T_{A}(|x|)\right)_{n} .
$$

Since $X$ is solid and $T_{A}(|x|) \in X$, these inequalities and (3.1) imply that $T_{B}(x) \in X$. Moreover, as $\|\cdot\|_{X}$ is a lattice norm it follows that

$$
\begin{aligned}
\left\|T_{B}(x)\right\|_{X} & =\left\|\left(\sum_{m=1}^{\infty} b_{n m} x_{m}\right)_{n=1}^{\infty}\right\|_{X} \leq\left\|\left(\sum_{m=1}^{\infty} a_{n m}\left|x_{m}\right|\right)_{n=1}^{\infty}\right\|_{X} \\
& =\left\|T_{A}(|x|)\right\|_{X} \leq\left\|T_{A}\right\|_{\mathrm{op}}\|x\|_{X},
\end{aligned}
$$


for each $x \in X$, where the stated series are actually finite sums. Hence, $\left\|T_{B}\right\|_{\mathrm{op}} \leq\left\|T_{A}\right\|_{\mathrm{op}}$ and the proof is complete.

Since the operator $T_{A}$ as given in Lemma 3.1 satisfies $T_{A} \geq 0$, it is clearly regular.

Corollary 3.2. Let $A=\left(a_{n m}\right)_{n, m=1}^{\infty}$ be a lower triangular matrix with non-negative real entries and $X \subseteq \mathbb{C}^{\mathbb{N}}$ be a Banach lattice sequence space such that $T_{A}(X) \subseteq X$. Let $B=\left(b_{n m}\right)_{n, m=1}^{\infty}$ be any matrix satisfying (3.2). Then the operator $T_{B} \in \mathcal{L}(X)$ is necessarily regular, that is, $T_{B} \in \mathcal{L}^{r}(X)$.

Proof. Define the non-negative real numbers $s_{n m}:=\left(\operatorname{Re} b_{n m}\right)^{+}, u_{n m}:=\left(\operatorname{Re} b_{n m}\right)^{-}, v_{n m}:=$ $\left(\operatorname{Im} b_{n m}\right)^{+}$and $w_{n m}:=\left(\operatorname{Im} b_{n m}\right)^{-}$for each $n, m \in \mathbb{N}$. Then $b_{n m}=\left(s_{n m}-u_{n m}\right)+i\left(v_{n m}-\right.$ $\left.w_{n m}\right)$ and $\left\{s_{n m}, u_{n m}, v_{n m}, w_{n m}\right\} \subseteq\left[0, a_{n m}\right]$ for $n, m \in \mathbb{N}$. Setting $S:=\left(s_{n m}\right)_{n, m=1}^{\infty}, U:=$ $\left(u_{n m}\right)_{n, m=1}^{\infty}, V:=\left(v_{n m}\right)_{n, m=1}^{\infty}$ and $W:=\left(w_{n m}\right)_{n, m=1}^{\infty}$ it is clear from the definition (3.1) that each operator $T_{S} \geq 0, T_{U} \geq 0, T_{V} \geq 0$ and $T_{W} \geq 0$ (in $X$ ) belongs to $\mathcal{L}(X)$; see Lemma 3.1. Since $T_{B}=\left(T_{S}-T_{U}\right)+i\left(T_{V}-T_{W}\right)$, it follows that $T_{B} \in \mathcal{L}^{r}(X)$.

Together with appropriate estimates, Corollary 3.2 will be the main ingredient required to establish (1.3) for $C$ (in place of $T$ ) when it acts in various classical Banach lattice sequence spaces $X$.

Let $\Sigma_{0}:=\{0\} \cup\left\{\frac{1}{n}: n \in \mathbb{N}\right\}$. We recall the formula for the inverses $(C-\lambda I)^{-1}$ : $\mathbb{C}^{\mathbb{N}} \longrightarrow \mathbb{C}^{\mathbb{N}}$ whenever $\lambda \in \mathbb{C} \backslash \Sigma_{0}$, [14, p.266]. Namely, for $n \in \mathbb{N}$ the $n$-th row of the lower triangular matrix determining $(C-\lambda I)^{-1}$ has the entries

$$
\frac{-1}{n \lambda^{2} \prod_{k=m}^{n}\left(1-\frac{1}{k \lambda}\right)}, \quad 1 \leq m<n, \quad \text { and } \quad \frac{n}{1-n \lambda}=\frac{1}{\left(\frac{1}{n}-\lambda\right)}, \quad m=n,
$$

with all other entries in row $n$ being 0 . We write

$$
(C-\lambda I)^{-1}=T_{D_{\lambda}}-\frac{1}{\lambda^{2}} T_{E_{\lambda}}
$$

where the diagonal matrix $D_{\lambda}=\left(d_{n m}(\lambda)\right)_{n, m=1}^{\infty}$ is given by

$$
d_{n n}(\lambda):=\frac{1}{\left(\frac{1}{n}-\lambda\right)} \quad \text { and } \quad d_{n m}(\lambda):=0 \quad \text { if } n \neq m .
$$

Setting $\gamma[\lambda]:=\operatorname{dist}\left(\lambda, \Sigma_{0}\right)>0$ it is routine to check that

$$
\left|d_{n n}(\lambda)\right| \leq \frac{1}{\gamma[\lambda]}, \quad n \in \mathbb{N}, \quad \lambda \in \mathbb{C} \backslash \Sigma_{0} .
$$

Moreover, $E_{\lambda}=\left(e_{n m}(\lambda)\right)_{n, m=1}^{\infty}$ is the lower triangular matrix given by $e_{1 m}(\lambda)=0$, for $m \in \mathbb{N}$, and for all $n \geq 2$ by

$$
e_{n m}(\lambda):=\left\{\begin{array}{cll}
\frac{1}{n \Pi_{k=m}^{n}\left(1-\frac{1}{k \lambda}\right)} & \text { if } & 1 \leq m<n \\
0 & \text { if } & m \geq n .
\end{array}\right.
$$

Lemma 3.3. Let $X \subseteq \mathbb{C}^{\mathbb{N}}$ be any Banach lattice sequence space. For each $\lambda \in \mathbb{C} \backslash \Sigma_{0}$ the diagonal operator $T_{D_{\lambda}}$, with $D_{\lambda}=\left(d_{n m}(\lambda)\right)_{n, m=1}^{\infty}$ given by (3.5), is regular in $X$, that is, $T_{D_{\lambda}} \in \mathcal{L}^{r}(X)$.

Proof. Fix $\lambda \notin \Sigma_{0}$ and let $A:=\frac{1}{\gamma[\lambda]} I$, where $I$ is the identity matrix in $\mathbb{C}^{\mathbb{N}}$, in which case $T_{A}(X) \subseteq X$ is clear. It follows from (3.6) that the matrix $B:=D_{\lambda}$ satisfies (3.2). Hence, the regularity of $T_{D_{\lambda}}$ in $X$ follows from Corollary 3.2 .

Remark 3.4. (i) Since any Banach lattice sequence space $X \subseteq \mathbb{C}^{\mathbb{N}}$ is a Banach function space over the $\sigma$-finite measure space $\left(\mathbb{N}, 2^{\mathbb{N}}, \mu\right)$, relative to counting measure $\mu$, and the function $n \longmapsto d_{n n}(\lambda)$ on $\mathbb{N}$ belongs to $L^{\infty}(\mu)$ by (3.6), the regularity of $T_{D_{\lambda}} \in \mathcal{L}(X)$ also follows from Proposition 2.1(i). 
(ii) For appropriate $X$ and $\lambda \notin \Sigma_{0}$, it is clear from (3.4) and Lemma 3.3 that the regularity of $(C-\lambda I)^{-1} \in \mathcal{L}(X)$ is completely determined by the matrix $E_{\lambda}$.

The following inequalities will be needed in the sequel. For $\alpha<1$ we refer to [14, Lemma 7] and for general $\alpha \in \mathbb{R}$ to [1, Lemma 3.2(i)].

Lemma 3.5. Let $\lambda \in \mathbb{C} \backslash \Sigma_{0}$ and set $\alpha:=\operatorname{Re}\left(\frac{1}{\lambda}\right)$. Then there exist positive constants $P(\alpha)$ and $Q(\alpha)$ such that

$$
\frac{P(\alpha)}{n^{\alpha}} \leq \prod_{k=1}^{n}\left|1-\frac{1}{k \lambda}\right| \leq \frac{Q(\alpha)}{n^{\alpha}}, \quad n \in \mathbb{N} .
$$

4. The Classical spaces $\ell^{p}, 1<p \leq \infty$, And $c_{0}$

For each $1<p \leq \infty$ let $C_{p} \in \mathcal{L}\left(\ell^{p}\right)$ denote the Cesàro operator as given by (1.4) when it is restricted to $\ell^{p}$. As a consequence of Hardy's inequality, [11, Theorem 326], it is known that $\left\|C_{p}\right\|_{\mathrm{op}}=p^{\prime}$, where $\frac{1}{p}+\frac{1}{p^{\prime}}=1$ (with $p^{\prime}:=1$ when $p=\infty$ ). Concerning the spectrum of $C_{p}$ we have

$$
\sigma\left(C_{p}\right)=\left\{\lambda \in \mathbb{C}:\left|\lambda-\frac{p^{\prime}}{2}\right| \leq \frac{p^{\prime}}{2}\right\}, \quad 1<p \leq \infty .
$$

Various proofs of (4.1) are known for $1<p<\infty$, [6], [12], [13], [15], [16]; see the discussion on p.268 of [6]. For the case $p=\infty$ we refer to [12, Theorem 4], for example.

Remark 4.1. For each $\lambda \neq 0$ set $\alpha:=\operatorname{Re}\left(\frac{1}{\lambda}\right)$. Then, for any $b>0$ we have

$$
\alpha<\frac{1}{b} \text { and only if }\left|\lambda-\frac{b}{2}\right|>\frac{b}{2} \text {. }
$$

The corresponding results for $\alpha>\frac{1}{b}$ and $\alpha=\frac{1}{b}$ also hold.

Proposition 4.2. For each $1<p<\infty$ the order spectrum of the positive operator $C_{p} \in \mathcal{L}\left(\ell^{p}\right)$ satisfies

$$
\sigma_{\mathrm{o}}\left(C_{p}\right)=\sigma\left(C_{p}\right)
$$

Proof. Via (1.2) it suffices to verify that $\rho\left(C_{p}\right) \subseteq \rho_{\mathrm{o}}\left(C_{p}\right)$.

With the notation of (3.4) and (3.7) it is shown on p.269 of [6], as a consequence of (3.8) in Lemma 3.5 above, that for every $\lambda \neq 0$ satisfying $\alpha:=\operatorname{Re}\left(\frac{1}{\lambda}\right)<1$ there exists a constant $\beta(\lambda)>0$ such that

$$
\left|e_{n m}(\lambda)\right| \leq \frac{\beta(\lambda)}{n^{1-\alpha} m^{\alpha}}, \quad 1 \leq m \leq n, \quad n \in \mathbb{N} .
$$

Set $B:=E_{\lambda}$ and let $A$ be the lower triangular matrix whose entries $a_{n m}(\lambda) \geq 0$ are given by the right-side of (4.3) for each $n \in \mathbb{N}$ and $1 \leq m \leq n$ (and 0 otherwise). According to (4.3) the matrices $A$ and $B$ satisfy (3.2). Let $X:=\ell^{p}$ for $p \in(1, \infty)$ fixed. Then Corollary 3.2 implies that $E_{\lambda}$ will be regular (i.e., $T_{E_{\lambda}} \in \mathcal{L}^{r}\left(\ell^{p}\right)$ ) whenever $T_{A}\left(\ell^{p}\right) \subseteq \ell^{p}$. Note that $T_{A} \in \mathcal{L}\left(\mathbb{C}^{\mathbb{N}}\right)$ is given by

$$
x \longmapsto \beta(\lambda)\left(\frac{1}{n^{1-\alpha}} \sum_{m=1}^{n} \frac{x_{m}}{m^{\alpha}}\right)_{n=1}^{\infty}:=\beta(\lambda) G_{\lambda}(x), \quad x \in \mathbb{C}^{\mathbb{N}} .
$$

So, if $\operatorname{Re}\left(\frac{1}{\lambda}\right)<1$, then (4.4) implies that $T_{A} \in \mathcal{L}\left(\ell^{p}\right)$ whenever $G_{\lambda}: \ell^{p} \longrightarrow \ell^{p}$ is continuous.

Let now $\lambda \in \rho\left(C_{p}\right)$, that is, $\left|\lambda-\frac{p^{\prime}}{2}\right|>\frac{p^{\prime}}{2}$. Then $\alpha:=\operatorname{Re}\left(\frac{1}{\lambda}\right)<\frac{1}{p^{\prime}}$, because of Remark 4.1. and hence, $(1-\alpha) p>1$. Then the Proposition on p.269 of [6] yields that indeed $G_{\lambda} \in \mathcal{L}\left(\ell^{p}\right)$. As noted above, this implies that $T_{E_{\lambda}} \in \mathcal{L}^{r}\left(\ell^{p}\right)$. Combined with (3.4) and Lemma 3.3 it follows that $\left(C_{p}-\lambda I\right)^{-1} \in \mathcal{L}^{r}\left(\ell^{p}\right)$, that is, $\lambda \in \rho_{\mathrm{o}}\left(C_{p}\right)$. This completes the proof of (4.2). 
Recall that $\left\|C_{\infty}\right\|_{\mathrm{op}}=1$ and, from (4.1) for $p=\infty$, that

$$
\sigma\left(C_{\infty}\right)=\left\{\lambda \in \mathbb{C}:\left|\lambda-\frac{1}{2}\right| \leq \frac{1}{2}\right\}
$$

Proposition 4.3. The order spectrum of the positive operator $C_{\infty} \in \mathcal{L}\left(\ell^{\infty}\right)$ satisfies

$$
\sigma_{\mathrm{o}}\left(C_{\infty}\right)=\sigma\left(C_{\infty}\right) .
$$

Proof. Again by (1.2) it suffices to prove that $\rho\left(C_{\infty}\right) \subseteq \rho_{\mathrm{o}}\left(C_{\infty}\right)$.

Fix $\lambda \in \rho\left(C_{\infty}\right)$. According to (4.5), for $b=1$ the condition in Remark 4.1 is satisfied with $\alpha:=\operatorname{Re}\left(\frac{1}{\lambda}\right)$. Hence, the inequalities (4.3) are valid and so $A:=\left(a_{n m}(\lambda)\right)_{n, m=1}^{\infty} \geq 0$ and $B:=E_{\lambda}$ can again be defined exactly as in the proof of Proposition 4.2. Then (3.2) is satisfied with $X:=\ell^{\infty}$. Arguing as in the proof of Proposition 4.2 (via Corollary 3.2) it remains to verify that $T_{A}: \ell^{\infty} \longrightarrow \ell^{\infty}$ is continuous, where $T_{A}$ is given by (4.4). To this effect, since $(1-\alpha)>0$ by Remark 4.1 , it follows that

$$
\sup _{n \in \mathbb{N}} \sum_{m=1}^{\infty}\left|a_{n m}(\lambda)\right|=\beta(\lambda) \sup _{n \in \mathbb{N}} \frac{1}{n^{1-\alpha}} \sum_{m=1}^{\infty} \frac{1}{m^{\alpha}}<\infty ;
$$

this has been verified on p.778 of [2] (put $w(n)=1$ there for all $n \in \mathbb{N}$ ) by considering each of the cases $\alpha<0, \alpha=0$ and $0<\alpha<1$ separately. But, condition (4.6) is known to imply that $T_{A} \in \mathcal{L}\left(\ell^{\infty}\right)$, [19, Ex.2, p.220]. The proof that $\lambda \in \rho_{\mathrm{o}}\left(C_{\infty}\right)$ is thereby complete.

To conclude this section we consider the Cesàro operator $C$, as given by (1.4), when it is restricted to $c_{0}$; denote this operator by $C_{0}$. It is shown in [12, Theorem 3], [14, that $\left\|C_{0}\right\|_{\text {op }}=1$ and

$$
\sigma\left(C_{0}\right)=\left\{\lambda \in \mathbb{C}:\left|\lambda-\frac{1}{2}\right| \leq \frac{1}{2}\right\} .
$$

Proposition 4.4. The order spectrum of the positive operator $C_{0} \in \mathcal{L}\left(c_{0}\right)$ satisfies

$$
\sigma_{\mathrm{o}}\left(C_{0}\right)=\sigma\left(C_{0}\right)
$$

Proof. Since (4.7) shows that $\sigma\left(C_{0}\right)=\sigma\left(C_{\infty}\right)$, the entire proof of Proposition 4.3 can be easily adapted (now for $X:=c_{0}$ and fixed $\lambda \in \rho\left(C_{0}\right)$ ), using the same notation, up to the stage where (4.6) is shown to be valid. In addition to the validity of (4.6) it is also true that

$$
\lim _{n \rightarrow \infty} a_{n m}(\lambda)=\frac{\beta(\lambda)}{m^{\alpha}} \lim _{n \rightarrow \infty} \frac{1}{n^{1-\alpha}}=0, \quad m \in \mathbb{N},
$$

because $\alpha:=\operatorname{Re}\left(\frac{1}{\lambda}\right)$ satisfies $(1-\alpha)>0$. The two conditions (4.6) and (4.8) together are known to imply that $T_{A} \in \mathcal{L}\left(c_{0}\right)$, [19, Theorem 4.51-C]. Again via Corollary 3.2 and Lemma 3.3 we can conclude that $T_{E_{\lambda}} \in \mathcal{L}^{r}\left(c_{0}\right)$ and hence, also $\left(C_{0}-\lambda I\right)^{-1}$ is regular on $c_{0}$.

5. The discrete Cesàro spaces $\operatorname{ces}(p), 1<p<\infty$, And $\operatorname{ces}(0)$

For $1<p<\infty$ the discrete Cesàro spaces are defined by

$$
\operatorname{ces}(p):=\left\{x \in \mathbb{C}^{\mathbb{N}}:\|x\|_{\operatorname{ces}(p)}:=\left(\sum_{n=1}^{\infty}\left(\frac{1}{n} \sum_{k=1}^{n}\left|x_{k}\right|\right)^{p}\right)^{1 / p}<\infty\right\} .
$$

In view of (1.4) we see that $\|x\|_{\operatorname{ces}(p)}=\|C(|x|)\|_{\ell^{p}}$ for $x \in \operatorname{ces}(p)$. It is known that each space $\operatorname{ces}(p), 1<p<\infty$, is a reflexive Banach lattice sequence space for the norm $\|\cdot\|_{\operatorname{ces}(p)}$ and the coordinatewise order. The spaces $\operatorname{ces}(p)$ have been thoroughly treated 
in [4]. According to Theorem 5.1 of [8] the restriction of $C$ (see (1.4) $)$ to $\operatorname{ces}(p)$, denoted here by $C_{(p)}$, is continuous with $\left\|C_{(p)}\right\|_{\text {op }}=p^{\prime}$ and

$$
\sigma\left(C_{(p)}\right)=\left\{\lambda \in \mathbb{C}:\left|\lambda-\frac{p^{\prime}}{2}\right| \leq \frac{p^{\prime}}{2}\right\}, \quad 1<p<\infty .
$$

Proposition 5.1. For each $1<p<\infty$ the order spectrum of the positive operator $C_{(p)} \in \mathcal{L}(\operatorname{ces}(\mathrm{p}))$ satisfies

$$
\sigma_{\mathrm{o}}\left(C_{(p)}\right)=\sigma\left(C_{(p)}\right)
$$

Proof. In view of (1.2) it suffices to verify that $\rho\left(C_{(p)}\right) \subseteq \rho_{\mathrm{o}}\left(C_{(p)}\right)$.

We decompose the set $\rho\left(C_{(p)}\right)$ into two disjoint parts, namely the set

$$
\rho_{1}:=\left\{\lambda \in \mathbb{C} \backslash\{0\}: \operatorname{Re}\left(\frac{1}{\lambda}\right) \leq 0\right\}=\{u \in \mathbb{C} \backslash\{0\}: \operatorname{Re}(u) \leq 0\}
$$

and its complement $\rho_{2}:=\rho\left(C_{(p)}\right) \backslash \rho_{1}$.

First fix $\lambda \in \rho_{1}$. Then $\lambda \notin \Sigma_{0}$ and so we may consider $E_{\lambda}=\left(e_{n m}(\lambda)\right)_{n, m=1}^{\infty}$ and $D_{\lambda}=\left(d_{n m}(\lambda)\right)_{n, m=1}^{\infty}$ as specified by (3.7) and (3.6), respectively. It is shown on p.72 of [8] that

$$
\left|e_{n m}(\lambda)\right| \leq \frac{1}{n}, \quad 1 \leq m<n, \quad n \in \mathbb{N} .
$$

Warning: In [8] the set $\mathbb{N}=\{0,1,2, \ldots\}$ is used rather than $\mathbb{N}=\{1,2,3, \ldots\}$ which is used here and so the inequalities from [8] are slightly different when they are stated here. Back to our proof, it is clear from (1.4) that the matrix $A=\left(c_{n m}\right)_{n, m=1}^{\infty}$ for the Cesàro operator $C$ is lower triangular with its $n$-th row, for each $n \in \mathbb{N}$, given by $c_{n m}:=\frac{1}{n}$ for $1 \leq m \leq n$ and $c_{n m}:=0$ for $m>n$. Setting $B:=E_{\lambda}$ it is clear from (5.4) that (3.2) is satisfied for the pair $A, B$ in the space $X:=\operatorname{ces}(p)$. Since $C_{(p)}=T_{A}: \operatorname{ces}(p) \longrightarrow \operatorname{ces}(p)$ is continuous, it follows from Corollary 3.2 that $T_{E_{\lambda}} \in \mathcal{L}^{r}(\operatorname{ces}(p))$ and hence, via Lemma 3.3 and (3.4), that also $\left(C_{(p)}-\lambda I\right)^{-1} \in \mathcal{L}^{r}(\operatorname{ces}(p))$.

Consider now the set $\rho_{2}$. From (5.1) it is routine to establish that a non-zero point $z \in \mathbb{C}$ belongs to $\sigma\left(C_{(p)}\right)$ if and only if $\operatorname{Re}\left(\frac{1}{z}\right) \geq \frac{1}{p^{\prime}}$. From the case of equality in Remark 4.1, it follows that $\rho_{2}=\bigcup_{0<\alpha<1 / p^{\prime}} \Gamma_{\alpha}$, where

$$
\Gamma_{\alpha}:=\left\{z \in \mathbb{C} \backslash\{0\}: \operatorname{Re}\left(\frac{1}{z}\right)=\alpha\right\}=\left\{z \in \mathbb{C} \backslash\{0\}:\left|z-\frac{1}{2 \alpha}\right|=\frac{1}{2 \alpha}\right\} .
$$

Fix a point $\lambda \in \rho_{2}$. Then there exists a unique number $\alpha \in\left(0, \frac{1}{p^{\prime}}\right)$ such that $\lambda \in \Gamma_{\alpha}$, namely $\alpha:=\operatorname{Re}\left(\frac{1}{\lambda}\right)$. In the notation of (3.7) it is shown on p.72 of [8] that

$$
\left|e_{n m}(\lambda)\right| \leq e_{n m}\left(\frac{1}{\alpha}\right), \quad n, m \in \mathbb{N} .
$$

Note that $e_{n m}\left(\frac{1}{\alpha}\right) \geq 0$ for all $n, m \in \mathbb{N}$ follows from (3.7) as $0<\alpha<\frac{1}{p^{\prime}}$ implies that $1-\frac{1}{k(1 / \alpha)}=\left(1-\frac{\alpha}{k}\right)>0$ for $m \leq k \leq n$. Setting $\widetilde{A}:=E_{1 / \alpha}$ and $\widetilde{B}:=E_{\lambda}$ it is clear from (5.6) that (3.2) is satisfied for the pair $\widetilde{A}, \widetilde{B}$ in place of $A, B$. Moreover, $\frac{1}{\alpha}>p^{\prime}$ implies that $\frac{1}{\alpha} \in \rho\left(C_{(p)}\right)$, that is, $\left(C_{(p)}-\frac{1}{\alpha} I\right)^{-1} \in \mathcal{L}(\operatorname{ces}(p))$. Since $T_{D_{1 / \alpha}} \in \mathcal{L}(\operatorname{ces}(p))$ by Lemma 3.3 (with $\frac{1}{\alpha}$ in place of $\lambda$ ), the identity $T_{E_{1 / \alpha}}=\alpha^{2}\left(T_{D_{1 / \alpha}}-\left(C_{(p)}-\frac{1}{\alpha} I\right)^{-1}\right)$ shows that $T_{\widetilde{A}} \in$ $\mathcal{L}(\operatorname{ces}(p))$. Hence, Corollary 3.2 can be applied to conclude that $T_{\widetilde{B}}=T_{E_{\lambda}} \in \mathcal{L}^{r}(\operatorname{ces}(p))$. It then follows from (3.4) and Lemma 3.3 that $\left(C_{(p)}-\lambda I\right)^{-1} \in \mathcal{L}^{r}(\operatorname{ces}(p))$. 
The remaining space to consider is $\operatorname{ces}(0):=\left\{x \in \mathbb{C}^{\mathbb{N}}: C(|x|) \in c_{0}\right\}$ equipped with the norm

$$
\|x\|_{\operatorname{ces}(0)}:=\|C(|x|)\|_{c_{0}}=\sup _{n \in \mathbb{N}} \frac{1}{n} \sum_{k=1}^{n}\left|x_{k}\right|, \quad x \in \operatorname{ces}(0) .
$$

It is a Banach lattice sequence space for the norm $\|\cdot\|_{\operatorname{ces}(0)}$ and the coordinatewise order. According to [8, Theorem 6.4], the restriction of $C$ (see (1.4) $)$ to ces(0), denoted here by $C_{(0)}$, is continuous with $\left\|C_{(0)}\right\|_{\mathrm{op}}=1$ and

$$
\sigma\left(C_{(0)}\right)=\left\{\lambda \in \mathbb{C}:\left|\lambda-\frac{1}{2}\right| \leq \frac{1}{2}\right\} .
$$

Proposition 5.2. The order spectrum of the positive operator $C_{(0)} \in \mathcal{L}(\operatorname{ces}(0))$ satisfies

$$
\sigma_{\mathrm{o}}\left(C_{(0)}\right)=\sigma\left(C_{(0)}\right) \text {. }
$$

Proof. As usual it suffices to show that $\rho\left(C_{(0)}\right) \subseteq \rho_{\mathrm{o}}\left(C_{(0)}\right)$.

Let the set $\rho_{1}$ be as in (5.3). For each $\alpha \in(0,1)$ let $\Gamma_{\alpha}$ be given by (5.5). Then (5.7) ensures that we have the disjoint partition $\rho\left(C_{(0)}\right)=\rho_{1} \cup \rho_{2}$ with $\rho_{2}:=\bigcup_{0<\alpha<1} \Gamma_{\alpha}$.

For any given point $\lambda \in \rho_{1}$ the estimates (5.4) are again valid (see [8, p.72]) and so the argument in the proof of Proposition 5.1 can be easily adapted ( now for $X:=\operatorname{ces}(0)$ ) to again show that $\left(C_{(0)}-\lambda I\right)^{-1} \in \mathcal{L}^{r}(\operatorname{ces}(0))$.

Fix now $\lambda \in \rho_{2}$. Then there exists a unique $\alpha \in(0,1)$ such that $\lambda \in \Gamma_{\alpha}$, namely $\alpha:=\operatorname{Re}\left(\frac{1}{\lambda}\right)$. Then $\operatorname{Re}\left(1-\frac{1}{k \lambda}\right)=\left(1-\frac{\alpha}{k}\right) \geq 0$ for $k \in \mathbb{N}$. Arguing as at the bottom of p.396 in [7, now with $x \in \operatorname{ces}(0)$ in place of $a \in \operatorname{ces}(2)$ there, it follows that the 1-st coordinate of $E_{\lambda}(x)$ is 0 and, for $n \geq 2$, that the $n$-th coordinate of $E_{\lambda}(x)$ satisfies

$$
\left|\left(E_{\lambda}(x)\right)_{n}\right| \leq\left(E_{1 / \alpha}(|x|)\right)_{n}, \quad x \in \operatorname{ces}(0) .
$$

Substituting $x:=\left(\delta_{r j}\right)_{j=1}^{\infty}$ into the previous estimates, for each $r \in \mathbb{N}$, yields (5.6). Since $0<\alpha<1$ implies that $\frac{1}{\alpha} \in \rho\left(C_{(0)}\right)$, the argument can be completed along the lines given in the proof of Proposition 5.1 to conclude that $\left(C_{(0)}-\lambda I\right)^{-1} \in \mathcal{L}^{r}(\operatorname{ces}(0))$. We again warn the reader that $\mathbb{N}=\{0,1,2, \ldots\}$ is used in [7].

Acknowledgement. The research of the first author (J. Bonet) was partially supported by the projects MTM2016-76647-P and GV Prometeo 2017/102 (Spain).

\section{REFERENCES}

1. A.A. Albanese, J. Bonet, W.J. Ricker, Spectrum and compactness of the Cesàro operator on weighted $\ell_{p}$ spaces, J. Aust. Math. Soc., 99 (2015), 287-314.

2. A.A. Albanese, J. Bonet, W.J. Ricker, Mean ergodicity and spectrum of the Cesàro operator on weighted $c_{0}$ spaces, Positivity, 20 (2016), 761-803.

3. W. Arendt, On the o-spectrum of regular operators and the spectrum of measures, Math. Z., 178 (1981), 271-287.

4. G. Bennett, Factorizing the classical inequalities, Mem. Amer. Math. Soc. 120 (Nr. 576) (1996), $1-130$.

5. F. F. Bonsall, J. Duncan, Complete Normed Algebras, Springer, Heidelberg-New York, 1973.

6. G.P. Curbera, W.J. Ricker, Spectrum of the Cesàro operator in $\ell^{p}$, Arch. Math. (Basel), 100 (2013), $267-271$

7. G.P. Curbera, W.J. Ricker, Solid extensions of the Cesàro operator on the Hardy space $H^{2}(\mathbb{D}), J$. Math. Anal. Appl., 407 (2013), 387-397.

8. G.P. Curbera, W.J. Ricker, Solid extensions of the Cesàro operator on $\ell^{p}$ and $c_{0}$, Integral Equ. Oper. Theory, 80 (2014), 61-77. 
9. B. de Pagter, W.J. Ricker, Algebras of multiplication operators in Banach function spaces, J. Oper. Theory, 42 (1999), 245-267.

10. D.H. Fremlin, Topological Riesz Spaces and Measure Theory, Cambridge University Press, Cambridge, 1974.

11. G.H. Hardy, J.E. Littlewood, G. Polya, Inequalities, 2nd ed., Cambridge University Press, Cambridge, 1964.

12. G. Leibowitz, Spectra of discrete Cesàro operators, Tamkang J. Math., 3 (1972), 123-132.

13. G. Leibowitz, Discrete Hausdorff transformations, Proc. Amer. Math. Soc., 38 (1973), 541-544.

14. J.B. Reade, On the spectrum of the Cesàro operator, Bull. London Math. Soc., 17 (1985), $263-267$.

15. B.E. Rhoades, Spectra of some Hausdorff matrices, Acta Sci. Math. (Szeged), 32 (1971), 91-100.

16. B.E. Rhoades, Generalized Hausdorff matrices bounded on $\ell^{p}$ and c, Acta Sci. Math. (Szeged), 43 (1981), 333-345.

17. H.H. Schaefer, Banach Lattices and Positive Operators, Springer, Berlin-Heidelberg-New York, 1974.

18. H.H. Schaefer, On the o-spectrum of order bounded operators, Math. Z., 154 (1977), 79-84.

19. A.E. Taylor, Introduction to Functional Analysis, Wiley, New York, 1958.

J. Bonet, Instituto Universitario de Matemática Pura y Aplicada iUmpa, Universitat Politècnica de València, 46071 Valencia, Spain

EMAIL:JBONET@MAT.UPV.ES

W.J. Ricker: Math.-Geogr. Fakultät, Kath. Universität Eichstätt-Ingolstadt, 85072 EichstätT, GERMANY

EMAIL: WERNER.RICKER@KU.DE 